\title{
Introduction : Les Vosges : vieux terroirs, nouveaux espaces
}

André Humbert

\section{(2) OpenEdition}

\section{Journals}

Édition électronique

URL : http://journals.openedition.org/rge/2338

DOI : $10.4000 /$ rge.2338

ISSN : $2108-6478$

Éditeur

Association des géographes de l'Est

Édition imprimée

Date de publication : 1 juin 2003

ISSN : 0035-3213

Référence électronique

André Humbert, «Introduction : Les Vosges : vieux terroirs, nouveaux espaces », Revue Géographique de l'Est [En ligne], vol 43 / 3 | 2003, mis en ligne le 24 novembre 2010, consulté le 25 septembre 2020 URL : http://journals.openedition.org/rge/2338 ; DOI : https://doi.org/10.4000/rge.2338

Ce document a été généré automatiquement le 25 septembre 2020.

Tous droits réservés 


\title{
Introduction : Les Vosges : vieux terroirs, nouveaux espaces
}

\author{
André Humbert
}

1 Les auteurs des quatre articles de ce numéro de la RGE ne prétendent pas présenter une géographie exhaustive du massif vosgien, ni même de sa partie méridionale, la plus anthropisée avec ses profondes vallées du versant lorrain et le vigoureux contact avec la plaine alsacienne. L'originalité de ces contributions réside plutôt dans le fait de souligner l'intérêt d'aborder l'étude d'une aire géographique à la fois par une géohistoire très "profonde» et par l'étude de manifestations très actuelles dont l'impact spatial est en train de s'inscrire sur le sol vosgien.

2 L'article présenté par le groupe de chercheurs alsaciens et coordonné par Dominique schwartz est un exemple intéressant de géographie historique appliquée à un paysage agraire fossilisé mais qui pose le problème de la colonisation du massif vosgien dans un secteur de transition entre le massif proprement dit et la plaine d'Alsace. Avec des méthodes qui ne sont pas couramment utilisées par les géographes, le groupe de chercheurs tente la reconstitution incertaine d'une action de mise en valeur agricole si lointaine que les vestiges retrouvés ne peuvent être interprétés que, de façon prudente, avec l'aide de procédés essentiellement archéologiques (fouille, datation au carbone 14). Les sources archivistiques sont mobilisées aussi mais elles sont bien maigres et, finalement, l'étymologie, aussi risquée soit-elle, est peut-être le meilleur garant de l'interprétation fonctionnelle de ces accumulations impressionnantes de pierres au sommet d'une colline sous-vosgienne. On aimerait plus de certitudes! Pourtant l'archéologue doit savoir se retenir, demeurer raisonnable au moment de construire un scénario plausible, car les informations sur lesquelles il établit «son histoire » sont clairsemées, discontinues, incertaines. Plus encore que dans d'autres secteurs des sciences humaines, l'interprétation des formes archéologiques exige prudence et modestie, et parfois renoncement provisoire, en attendant que de nouvelles pièces puissent être versées au dossier : ces nouveaux éléments peuvent provenir d'analyses plus fines sur l'espace étudié mais aussi de comparaisons avec des sites voisins en cours d'exploration. Peut-être aussi faudra-t-il aller regarder ailleurs, dans des régions plus 
lointaines où des phénomènes semblables ont été observés. C'est le support géologique qui, sans doute, est susceptible de fournir la clé de l'explication globale. Partout où les substrats calcaires facilement délitables ou fractionnables servent d'assise à un terroir, les hommes ont dû se débarrasser des pierres d'une façon ou d'une l'autre, le plus souvent en les accumulant sur les bordures, puis au centre même des parcelles lorsque l'érosion du sol arable faisait "pousser" les pierres au point d'obliger les paysans à choisir les solutions les moins dévoreuses de temps et de labeur. Les exemples de ces formes sont innombrables dans les régions de "croûte" si nombreuses autour de la Méditerranée; mais il en existe aussi de beaucoup plus proches, sur le même support précisément que celui du terroir alsacien, c'est-à-dire du Muschelkalk, juste de l'autre côté des Vosges, sur le plateau du piémont occidental où des actions systématiques d'épierrement, probablement encouragées par des édits des ducs de Lorraine, au début du XVII siècle, ont donné naissance, à une époque plus récente que celle du Bickenberg alsacien, à un curieux paysage « bocager » né de l'installation de végétation ligneuse au long des cordons de pierres accumulées sur les limites parcellaires d'une structure agraire d'openfield. Peut-être une partie des réponses aux questions que se posent les archéologues du Bickenberg se trouvent-elles précisément au-delà de la ligne bleue des Vosges, vers le nord-ouest, entre Sarrebourg et Blamont.

3 C'est une rétrospective beaucoup plus proche que nous propose Simon Edelblutte mais qui correspond à une phase décisive de la mise en place des paysages vosgiens actuels. En effet, l'étude très fine de la vallée bressaude que présente l'auteur nous permet de démêler les fils d'un écheveau complexe de formes tressées au fond d'une des vallées les plus emblématiques du massif. Les images sur lesquelles il fonde ses observations le conduisent à dresser un état des lieux d'où surgissent des questions, des hypothèses, des pistes de recherche et des conclusions. Cette démarche rigoureusement géographique lui permet de montrer à quel point l'activité industrielle qui a d'abord occupé des paysans-ouvriers au XVIII ${ }^{\mathrm{e}}$ siècle avant de les transformer en ouvrierspaysans des filatures et tissages du XIX ${ }^{e}$, est aujourd'hui encore un des piliers de l'économie, avant même le tourisme qui assure pourtant la renommée lointaine de la petite ville. Si les statistiques officielles de l'emploi confirment cette originalité bressaude, ce sont bien les objets géographiques consacrés à l'industrie avec leur architecture datée, leur concentration à la périphérie du vieux foyer d'habitat qui orientent la réflexion sur ce qui est l'objet propre de la géographie humaine, c'est-àdire l'aménagement par l'homme et pour l'homme de «la face de la terre ». S. Edelblutte en fait la démonstration en prenant appui sur des documents aériens qui lui offrent un regard global indispensable pour saisir les « emboitements d'échelle » si utiles à la compréhension des organisations spatiales. A partir de ce complexe de faciès paysagers, d'ensembles morphologiquement cohérents et d'objets fonctionnellement significatifs, il sait exprimer l'ordre fonctionnel, l'organisation actuelle de cet espace montagnard, mais aussi exhumer certains systèmes territoriaux révolus grâce aux vestiges qu'ils ont laissés incrustés dans les paysages actuels; ce sont autant de marqueurs précieux que le géographe sait exploiter en archéologue, car en dépit des différences entre les deux disciplines, il s'agit bien, dans l'un et l'autre cas d'utiliser des témoins fossilisés, qu'ils le soient depuis cinquante ans, comme à la Bresse, ou depuis cinq siècles sur le Bickenberg.

4 Les deux derniers articles de ce numéro étudient des phénomènes vosgiens incontestablement plus contemporains puisqu'il s'agit de manifestations qui parfois n'ont pas encore produit tous leurs effets spatiaux. En effet, il s'agit bien de mutations 
en cours qui concernent les périphéries plus ou moins proches de quelques villes vosgiennes.

5 Le cadre spatial choisi par Vincent Bertrand pour son étude, celui du Parc Naturel Régional des Ballons des Vosges peut apparaître comme un peu circonstanciel. Si cela est peut-être le cas, ce choix permet, en tout cas à l'auteur de cet article de souligner une apparente contradiction entre la vocation «naturelle» d'un territoire et son caractère urbain déclaré par les statistiques. En effet, dans les limites du Parc Naturel existeraient 30 communes que l'INSEE classe parmi les communes urbaines. V. Bertrand soulève là un problème qui intéresse le géographe au premier chef et qu'il rencontre chaque fois qu'il aborde un territoire par l'entrée statistique. Que sont ces trente «villes » vosgiennes que le Parc intégrerait, contre toute logique, dans son territoire? Si l'on exclut les "villes-portes ", ces villes sont en réalité, le plus souvent, des bourgs modestes au cœur d'un vaste territoire rural dans lequel sont disséminés des hameaux de toute taille dont la population cumulée fait franchir à la commune le seuil des 2000 habitants et l'élève ainsi au rang de ville statistique. L'espace du Parc Naturel des Ballons est donc bien, pour l'essentiel, un espace rural mais dans lequel V. Bertrand décèle l'influence des villes qui s'insinuent au long des vallées et se manifeste par des mouvements pendulaires qui conduisent employés et chalands vers leurs lieux de travail ou d'achats urbains. L'auteur illustre ce phénomène, aujourd'hui somme toute fréquent autour des villes françaises, par l'exemple de la commune, chef-lieu de canton de Provenchères-sur-Fave que la rurbanisation - ou la périurbanisation déodatienne a vivifiée au point d'en faire un centre relais pour les campagnes de la partie nord du bassin de Saint-Dié adossées aux terres alsaciennes. Si, aborder la rurbanisation par l'observation statistique de l'emploi est une approche qui ne saurait être négligée, bien d'autres manifestations plus spatiales du phénomène méritent d'être prises en compte pour apprécier l'irruption - ou la lente diffusion - des formes urbaines dans les campagnes. C'est ce remodelage morphologique qu'évoque discrètement l'auteur avec l'évolution des fonctions de l'ancien bourg de Provenchères.

6 C'est aussi des villes vosgiennes dont il s'agit dans l'article présenté par Colette RenardGrandmontagne. Des deux villes principales, Épinal et Saint-Dié autour desquelles se manifestent des phénomènes devenus ubiquistes, au moins dans tous les pays développés. En effet, depuis une quarantaine d'années les périphéries urbaines ont été envahies non seulement par diverses formes d'habitat moderne mais aussi par des bourgeonnements sécrétés par des activités dont beaucoup étaient autrefois localisées à l'intérieur de la ville elle-même. Quelle originalité présentent les Vosges ? L'auteur qui a recensé de manière exhaustive toutes les zones d'activités établies dans le département, souligne, grâce à la carte, le déséquilibre de la répartition de ces espaces consacrés aux activités, qu'elles soient industrielles ou commerciales, au profit de la partie orientale, c'est-à-dire de la montagne, ou plus précisément des villes établies dans les grandes vallées qui pénètrent dans le massif. Si S. Edelblutte a insisté sur l'importance de l'industrie, aujourd'hui encore, dans une petite ville de l'intérieur, autrefois fortement marquée par le textile, ce sont les deux villes installées au débouché de la montagne qui intéressent $C$. Renard-Grandmontagne. Partant d'un état des lieux - spatial et statistique - l'auteur ne se contente pas de dresser un inventaire, elle analyse la progression des formes et leur contenu fonctionnel pour tenter de comprendre par quels mécanismes économiques, historiques, politiques les zones d'activités se sont progressivement mises en place autour d'Épinal et de Saint-Dié. Ses conclusions, tout en nuances, révèlent que, si le développement des zones d'activités 
périurbaines est un phénomène généralisé, les villes vosgiennes sont concernées selon des modalités locales qui ont orienté de façon particulière à la fois les choix spatiaux et la chronologie. Le géographe remplit ici sa fonction qui est de déceler et d'expliquer des identités territoriales occultées trop souvent par l'exposé de quelques grands mécanismes économiques de portée mondiale.

7 La géographie vosgienne présentée dans ce numéro de la RGE est certes très partielle, parfois ponctuelle; elle est toujours historique, c'est-à-dire génétique, car même les strates les plus récentes des paysages gardent les vestiges des épisodes passés, parfois comme des corps devenus étrangers et inutiles, parfois, au contraire, en les adaptant à de nouvelles fonctions après en avoir altéré plus ou moins la physionomie. Bien d'autres aspects de cette montagne vivante auraient pu trouver leur place ici. Ce constat est aussi une invitation aux chercheurs des deux versants de la montagne vosgienne.

\section{AUTEUR}

\section{ANDRÉ HUMBERT}

CERPA / Université Nancy 2 - BP 33-97 - 54015 NANCY cedex - Andre.Humbert@univ-nancy2.fr 